\title{
Analysis of the Differences of Emoticons in Intercultural Online Communication
}

\author{
Hanyu $\mathrm{Cao}^{1, *}$ \\ ${ }^{1}$ College of Liberal Arts, Journalism and Communication, Ocean University of China, Qingdao, Shandong \\ 266100, China \\ *Corresponding author.Email:18140002002@stu.ouc.edu.cn
}

\begin{abstract}
With the popularization of the online social media, the communication of various cultures in the world is becoming closer, and the use of emoticons is becoming much more frequent. Among them, there are not only emoji, a traditional emoticon, but also Animoji and "Biaoqingbao", which have sprung up in recent years. All kinds of emoticons are transmitted in the online world, but the differences then come forth obviously. This paper uses text analysis, comparing emoticons used in various countries, proposes to conclude how emoticons differ on the social media including the production, usage and comprehension. Because of the differences of way of thinking, language mechanism, culture and social media preference, Easterners and Westerners prefer using different forms of emoticons in different ways, and they also understand the meanings differently. Hence, for the sake of better intercultural online communication, people have to respect others from different cultures of both nations and social media, as try to use acknowledged and simple emoticons.
\end{abstract}

Keywords: Intercultural communication, Emoticons, Social media, Online communication.

\section{INTRODUCTION}

Emoticon is a communication method that people use when communicating on the Internet to express and vent specific or personal emotions. It used to be consisted of simple punctuation marks, then derived to Emojis, Animojis, "Biaoqingbao" and so on. Nowadays, emoticon is widely used on social media like Twitter, Facebook, Kakaotalk and WeChat.

Research on emoticon found that factors such as knowledge background, culture and gender could affect the position, frequency, and sentiments of emoticons in Instant Massaging[1], [2], [3]. These factors may have similar effects on emoticon usage in intercultural online communication. Due to the factors like inconsistent understanding of some emoticons and the cultural difference, people may have a bad intercultural communicating experience. That is particularly acute between the West and the East. In order to diminish the obstacles in intercultural online communication, The author will focus on the differences of the emoticons used in the Eastern and the Western social media. This paper will compare the differences between emoticons on different social media in the East and the West, and analyzes how they affect the online communication between Easterners and Westerners, aiming to find the reasons and solutions to misunderstanding. Though there are many researches on the emoticons, few of them consider the intercultural communication, this paper will fill the gaps and provide suggestions for the better intercultural online communication on social media.

\section{OVERVIEW OF EMOTICONS}

\subsection{Development of Emoticons}

In recent years, with the development of the Internet, emoticon has emerged. The first online emoticons were created in 1982 by Professor Fallman of Carnegie Mellon University in the United States. This is an abstract emoticon obtained by rotating 90 degrees counter-clockwise with the combination of characters in ASCII code, which can express simple emotions. The Internet gradually entered Asia and became popular. Correspondingly, emoticons, originally only popular in Europe, are also popular in Asia. In Asia, 
emoticons have also developed further, and led a new trend, which has spread back to European emoticons. Nowadays, with the emergence of various social media, their official emojis have become popular, the emojis in Skype is a typical representative. As the social media develop, emoticons have entered a new stage of development. Popular stars, anime characters and animals are chosen as the original material, accompanied by humorous words, made into Animojis or "Biaoqingbao" which has been popular for years around the world, but still mainly used on Eastern social media.

\subsection{Emoticon Culture Under Intercultural Online Communication}

With the globalization and the popularization of the Internet, all cultures are inevitably influenced by other cultures, so are emoticons. When people communicate online, using emoticons can be regarded as a new type of nonverbal communication behavior. Recently, it has already become an important way of expression and gotten widely used. In intercultural online exchanges, Eastern and Western cultures blend and influence each other. Emotional originated in Europe, but prevailed in Asia, and evolved different characteristics, ending up with great difference between the West and the East.At the same time, the wide use of emoticons has also derived many Internet terms, such as "fight with emoticons" which means people send emoticons endlessly during communication.

\section{DIFFERENCES OF EMOTICONS ON EASTERN AND WESTERN SOCIAL MEDIA}

Social media from different countries have different systems of emoticons. And people of different countries attach different functions on them. In this part, the paper will have case-study on two social media: WeChat for the Orientals, Twitter for the Westerners, and conclude the differences in the comparison.

\subsection{Emoticons on WeChat}

WeChat is now popular in the East, especially in Southeast Asia, its iconic emojis are Soybean Emojis, which are similar to other round facial emojis and are able to represent the emojis on Eastern social media like Sina Weibo, Kakaotalk. They are basically forward-facing and more realistic. But WeChat is different from other media platforms in that there are no restrictions on selfmade emoticons. The Eastern media seem to have more diverse forms including pictures, GIF, and even derivatives of Intellectual Property(IP) characters. On WeChat, thousands of self-made Animojis of IP characters can be found. Artists are free to upload Animojis and all other users can download, though sometimes they have to pay a little. Everyday, more than 50 series of Animojis hit the shelves, that data dramatically rise to around 110 before the Spring Festival 2020. With such a sufficient database of emoticons, Oriental social media are able to serve users in other aspects. Sina Weibo and QQ, they even could recommend intelligently suitable Animojis according to the text when users chat or post.

Except the Animojis, there is another kind of self-made emoticon called "Biaoqingbao", which are always made by normal people out of interest. "Biaoqingbao" is widely used in the East, especially in Asian countries like China. People often use a variety of "Biaoqingbao" in online communication to replace words. Unlike Animojis, "Biaoqingbao" is always secondary creation of movies and televisions in picture form. People intercept clips with funny faces or lines and add words on it. As for the preference of WeChat emotion usage, Orientals seem to be the same. They prefer Animojis for social function, which shows the complete action while the static ones must be accompanied by explanatory text. Among all these Animojis, the characters are usually cute animals like cats. Cute Animoji is like the lubricant in social media interpersonal communication. Even for serious dialogue, a cute Animoji can bridge the gap in a friendly way, making people get closer, which is necessary to improve interpersonal communication.

Besides, Easterners often choose emoticons having positive meanings when chatting with friends in secondary or cross-generation relationships as a way to bind them closer.[4] In the top 10 emojis used in WeChat, "grin" is the most popular at $43.88 \%$ with a higher frequency of use, while the only negative emojis "sob" only takes up $3.55 \%$. [5] WeChat imports some emojis like "face with tears of joy" "flushed face", because those emojis are already widely used on other social media and WeChat wants to addict users with them. However, the emojis mainly turned to convey more deep meanings in the East. For example, the emojis of "smile" is used in the Oriental social media to express angry ironically. The purpose is to express 
discontent, while the Western people express intend to humor or friendship.[6]

\subsection{Emoticons on Twitter}

Twitter is one of the most common social media, like those on WhatsApp or other Western social media, most of the emojis on Twitter are exaggerated and flat, holding less details than WeChat. Twitter is a social platform mainly for sending short messages, so the emojis designed by Twitter are very simple, with almost no shadows, borders and other details. In addition, Twitter's emoji is basically a side-view face, which is not seen in the WeChat emoji design. When interpreting the specific meaning, most of the emojis need to be rotated 45 degrees counterclockwise such as "rolling on the floor laughing". In fact, Twitter is widely used in the world, its Twimojis are divided into 8 types containing almost everything like smileys and people in all colors, flags, occupations, animals and food. However, the Soybean Emojis are mostly smileys and ordinary stuff like coffee, beer and even red pocket, consisting a database of only 109 emojis. Western social media also attempt to supply various forms of emoticons except the traditional tiny, round emoji faces. For instance, Skype imported a series of mojis which look like short videos in 2015 while users can also search emoticons on Twitter, not only for Twimojis, but also for GIFs which are clarified according to emotions like anger, joy and so on.

Western people often use emoticons as an illustration or a picture of a text, but seldom use it individually in daily communication. Obviously, the western emojis emphasize more explanatory function than social function, while the eastern "Biaoqingbao" emphasizes the opposite. Westerners use emoticons to improve their content when communicating online instead of totally relying on them. As a result, although Twitter allows users to comment with pictures or GIFs, the emoticons always follow behind the opinions to emphasize their feelings. Besides, when posting, Westerners are used to decorating the text with symbol emojis. They would be put at the beginning of the sentence as sequence numbers or between sentences as filed separators.

On Western social media, specific emoji orders can refer to some other things, like the set of heart emojis with color order red, white, blue could represent colors for a sports team or a country flag. This requires communicators have the same cultural perception. Comparing with WeChat users derive the deep meanings of emojis in a negative way, Twitter users do that much more positively, close to the original meanings. For example, "hugging face" represents rejection or sarcasm on WeChat but is used on Twitter to convey their excitement when the emoji "heart" is just not enough.

\section{REASONS FOR THE DIFFERENCES OF EMOTICONS BETWEEN THE EAST AND WEST}

\subsection{Different Ways of Thinking Influence the Formats of Emoticons}

The way of thinking between the East and West has been different for years and extends to the online world. Westerners' way of thinking is more direct and straightforward. For example, the content will be clearly stated in the opening sentence, and there will be less unimportant and declarative content. They pay attention to the analysis of speculative rationality. But the way of thinking of Oriental people is tortuous, characterized by integrity and intuition. [7] They are used to starting from the description of the external environment and finally pointing out the substance. Conversation often develops from secondary to primary, from relevant information to main topics. As a result, the western emoticons are always simple with short keywords while the words usually hide deeply in Eastern emoticons like "Biaoqingbao", which takes seconds to get the true meaning.

\subsection{Differences in Language Mechanisms and Preference of Social Media Influence the Usage of Emotions}

In intercultural communication, the mechanisms of different languages constitute a significant difference in the emoticon mechanism between the two. Western languages, take English as an example. When using English, people often use long sentences, clauses and pronouns, and this category is mostly passive. It forces users to read a complete sentence to understand the meaning with less ambiguity. So Westerners often take emoticons as a addendum to their words, adding emphasis to their emotions. Eastern languages such as Chinese and Japanese are highly subjective and attach great importance to the expression of their meaning. Unlike English, Eastern languages do not pay much attention to form and rigorous syntactic structure, 
and the role of verbs is not so prominent. In this case, the language is also more specific [8] This character also shows on the eastern emoticons, which contains strong emotions and can be used individually.

Moreover, different social media have different functions, which effect the emotion usage. Westerners and Easterners have different favor of social media, but they all rely on these media when communicating online, no matter it is a public or a private platform. So, the functions the social media provide play a role in the cultivation of users' emoticon using habit. For example, Oriental WeChat facilitates people to use emoticons they like freely, which also pushes the development of "Biaoqingbao". As for the western apps like Skype or Whatsapp, they limit the range, type of emoticons they can use, and most of them are official. People have to store thousands of emoticons in their phone albums. It is so troublesome that Westerners prefer to use a group of official emojis to express their feelings.

\subsection{Differences in Values and Cultures Influence the Understanding of Emoticons}

Hoffstead' s individualism-collectivism theory holds that, "In an individualistic society, interpersonal relationships are loose and everyone takes care of himself and his family individually; on the contrary, in a collectivistic society, people are united from birth with powerful and cohesive internal groups, which provide lifelong support for these loyal members." [9] In the western countries like the United States, people emphasizes that individualism is higher than collectivism. People are individual-oriented, leading to a unstable relationship between people, they seldom pay attention to interpersonal relationships. This is also the obvious reason why Westerners use simple emoji to express. The Oriental countries, such as Japan, Korea and China, have the cultural tradition of emphasizing interpersonal relationship. They pursue harmony, and collectivism is higher for them. Besides, Eastern culture is introverted and reserved, so even if people in the East are angry, they tend to hide their anger with emojis of "smile," "grin," and "embarrassment." It has already become a common use on eastern social media, but requires western communicators to feel and understand for himself.

\section{WAYS TO REDUCE BARRIERS TO INTERCULTURAL ONLINE COMMUNICATION}

\subsection{Learning About Others' Cultures}

Any society has its specific social and cultural norms which restrict people's behavior and thinking. In order to avoid this communication barrier thoroughly, the communicators should begin with understand others' cultures. Only by respecting the customs of different countries can we understand the country's cultural and social development. Understanding different cultures can also avoid misunderstandings and conflicts due to cultural differences. For example, "dog" is a derogatory word in Chinese. Idioms like "a fox friend, a dog friend" refer to a bad friend. While in English, "dog" is like the most loyal friends. It is used to describe the other party with praise, such as "you are a lucky dog." If people can understand these linguistic and cultural differences, misunderstandings and ambiguities will rarely occur in communication.[10]

Likewise, social media also develop their own culture mechanisms. When we want to fit into a foreign online community, people must learn about its idiomatic usage of emoticons and catchphrases. For instance, on Eastern social media especially Chinese ones, there is a new trend of partial-tone words, like "pear" sounds like "pressure" in Chinese, so people tend to use emoji "pear" to represent pressure on social media. While on Twitter, Facebook or other global social media, we need to pay attention to the taboos like "okay" emoji which means "white supremacy" but ignored in the East.

\subsection{Respecting Different Cultures and Expressing Carefully}

On the social media, it is difficult to fully understand the culture of different countries. That often happens between close friends who are very familiar with each other. It is only possible to understand the popular views, which are not entirely accurate. There are already a lot of transnational quarrels caused by different cultural understandings. For instance, in 2016, some people from the same Chines online community flocked into Facebook and had a fierce fight with foreign users, and "Biaoqingbao" was one of their major weapons. 
To avoid conflicts, people would better calm down and respect the way how others use emoticons. But in Western culture, it is just a symbol of joy, kindness, and politeness, so Westerners always use it to say hello on social media, which has gained more recognition worldwide. In this case, when being greeted by a "smile", it is much better to think twice before insulting and respond with polite language.

\subsection{Trying to Use Simple Emoticons}

When using the same emoticon, Orientals often give it deeper meanings, but that hardly exist in the West. Due to the different understanding of the same emoticons between the East and the West, there are often unnecessary misunderstandings in intercultural communication. Therefore, the best solution is to reduce the use of emoticons with implicit meanings such as metaphors, especially "Biaoqingbao" with ethnic or group cultural specificities. People should use easy-to-understand, non-figurative emoticons on social media, such as official emoticons.

\section{CONCLUSION}

Emoticons are extremely important during the intercultural online communication, which are widely used by both Easterners and Westerners and derive more characters. Due to a lot of factors like differences to the way of thinking, language mechanism, preference of social media and values, the forms, production, usage and understanding of emoticons varied from the West to the East. Hence, when have intercultural online communication, people should be more cautious and try their best to overcome the obstacles. This article focuses on the overall differences of social media between the Eastern and the Western emojis, while ignoring the individual differences between the East and the West. Therefore, in future research, researchers can focus on user behavior analysis when using various expressions.

\section{AUTHORS' CONTRIBUTIONS}

This paper is independently completed by Hanyu Cao.

\section{ACKNOWLEDGMENTS}

Thanks are due to my professor Mengyu Li, my supervisor, for her assistance with my paper and valuable discussion. I am deeply grateful for her help of the completion of my paper. Without her illuminating and consistent instruction, this paper could not have reached its present form.

High tribute to Ms. Hayley, whose profound knowledge of media helps me to have a better understanding of the emotion phenomenon and find the best research method.

Special thanks should go to my friends and parents for their continuous support and encouragement.

\section{REFERENCES}

[1] Garrison, A., et al.: Conventional faces: emoticons in instant messaging discourse. Comput. Compos. 28(2), 112-125 (2011)

[2] Hautasaari, A.M., Yamashita, N., Gao, G.: Maybe it was a joke: emotion detection in text-only communication by non-native English speakers. In: Proceedings of the $32^{\text {nd }}$ Annual ACM Conference on Human Factors in Computing Systems. ACM (2014)

[3] Ogletree, S.M., Fancher, J., Gill, S.: Gender and texting: masculinity, femininity, and gender role ideology. Comput. Hum. Behav. 37, 49-55 (2014)

[4] An J., Li T., Teng Y., Zhang P. Factors Influencing Emoji Usage in Smartphone Mediated Communications. In: Chowdhury G., McLeod J., Gillet V., Willett P. (eds) Transforming Digital Worlds, 423-428 (2018)

[5] Liu Xuetai, Ba Zhichao, Li Yang, Li Gang. Research on the Differences and Semantics of Emoticons in the Process of Social Media Group Information Exchange-Taking WeChat Groups as an Example[J]. Modern Intelligence, 2019, 39(10): 3-14.

[6] Captain Patrick M. Milott.(2018). Emojis and Emoticons in Court. The Reporter,3,61-67

[7] Shang, Y.W., Su Z.Q., \& Han, Y..(2019) A Study on the Differences of Emotional Symbols in Intercultural Communication. New Media Research,7,5-8

[8] Li, K.(2005).Looking at the differences between English and Chinese expressions from the comparison of Chinese and Western ways of thinking. Journal of Xinjiang Normal University (Philosophy and Social Sciences Edition), 8,211-215 
[9] Peng, S.Y.(2004).Hofstad's Cultural Value Theory and Research Method. Journal of Military Foreign Languages College, 1,96-99

[10] Bousfield, D. Impoliteness in Interaction[M]. Amsterdam/Philadelphia: John Benjamins Publishing Company, 2008:45 